\title{
Advances in Gas Hydrate Thermodynamics and Transport Properties
}

\author{
Angelo Lucia, ${ }^{1}$ Jae W. Lee, ${ }^{2}$ and Costas Tsouris ${ }^{3}$ \\ ${ }^{1}$ Department of Chemical Engineering, University of Rhode Island, Kingston, RI 02881, USA \\ ${ }^{2}$ Department of Chemical Engineering, City College of New York, New York, NY 10031, USA \\ ${ }^{3}$ Environmental Sciences Division, Oak Ridge National Laboratory, Oak Ridge, TN 37831-6181, USA
}

Correspondence should be addressed to Angelo Lucia, lucia@egr.uri.edu

Received 2 March 2010; Accepted 2 March 2010

Copyright (c) 2010 Angelo Lucia et al. This is an open access article distributed under the Creative Commons Attribution License, which permits unrestricted use, distribution, and reproduction in any medium, provided the original work is properly cited.

Gas hydrates or clathrate hydrates are important in many applications in science and engineering - in flow assurance, in geological and deep ocean sedimentary carbon storage, hydrogen transportation and storage, methane and natural gas production, and others. This special issue of the Journal of Thermodynamics, entitled Advances in Gas Hydrate Thermodynamic and Transport Properties, is intended to give the reader an appreciation for recent developments and current research activities related to gas hydrates. There is a collection of eight papers in this special issue, ranging in topic from experimental phase equilibrium to molecular simulations of clathrate structures and stability, multiscale modeling and simulation of phase behavior and reservoirs, to observational investigations of natural deposits of hydrates.

The first paper is a collaborative contribution from Prairie View A \& M University, Georgia Institute of Technology, and Oak Ridge National Laboratory by Drs. Gabitto and Tsouris and provides a short review on the physical properties of pure and sediment-bearing gas hydrates. The authors give an excellent overview of the fundamental structures (sI, sII, and $\mathrm{sH}$ ) of gas hydrates as well as discussing the morphology and physical property differences between pure and sediment-bearing gas hydrates. Gabitto and Tsouris note that all three gas hydrate structures, when completely occupied by gas, have essentially the mechanical properties of ice with the exception of thermal expansivity and thermal conductivity. Moreover, they discuss the strong interaction between hydrates and sediments that affects the morphology and properties of both.

The next article is from a research group from Osaka University in Japan led by Dr. Shunsuke Hashimoto. Hashimoto et al. present a study of the thermodynamic properties of mixed gas hydrates with tetra n-butyl ammonium salt additives, which have great potential in hydrogen transportation and storage at much lower pressures than pure hydrogen hydrates. This class of hydrates, called semi-clathrates, consists of ammonium cations and anions incorporated directly into the water cage. The group from Osaka University has specifically studied the stability properties of tetra nbutyl ammonium bromide (TBAB) using phase equilibrium experiments and Raman spectroscopy and concluded that it is possible to obtain reversible storage and release of hydrogen without destroying the semi-clathrate structure of TBAB hydrate cages.

Following this, there are three papers affiliated with the group of Professor JW Lee from City College of New York. The first of these papers is by Yedlapalli et al. and is a collaborative effort between CCNY and Texas A \& $M$ University. It centers on the use of $a b$ initio quantum chemistry simulations to build understanding of the stability of hydrogen clathrates with and without tetrahydrofuran (THF). Yedlapalli et al. conduct detailed $a b$ initio quantum chemistry calculations using the PCGAMESS 7.0 computer package to study single and double occupancy hydrogen clathrates using a double cavity model. The authors conclude from their study that single occupancy is slightly more favorable (stable) than double occupancy for the pure hydrogen clathrate and the $\mathrm{H}_{2}+$ THF binary clathrate.

In the fourth paper, Jatkar et al. use molecular dynamics simulations to determine reference chemical potentials for hydrates. A new model which includes lattice distortion of the water cage by the guest molecule is considered. 
Molecular dynamics simulations were conducted using the TIP4P water model to determine the difference between the chemical potential of water and a theoretically empty cage at the reference state for sII gas hydrates. In their work, Jatkar et al. provide understanding of lattice distortion and related temperature effects, which are important in enthalpy calculations and show that there are still difficulties in calculating accurate reference chemical potentials for gas hydrates.

The fifth paper is a contribution by Jones et al. on the determination of the effect that isotopes have on the eutectic temperature and melting temperature of watertetrahydrofuran (THF) gas hydrates. In this work, the authors study the effect of isotopic substitution on the eutectic and melting temperature of THF hydrates. In particular, Jones et al. use differential scanning calorimetry to study the effects of replacing water with deuterated water. The authors report a 3-to-4 degree Kelvins increase in the melting temperature of THF-water hydrates and a similar increase in the eutectic temperature. On the other hand, enclathration of deuterated THF causes a smaller but reverse effect in melting temperature but a slight increase in eutectic temperature. The authors attribute these changes to the motion of the guest molecules and recommend further studies to build better understanding of the effects of the motion of guest molecules on properties such as thermal conductivity.

The next paper is a contribution from the University of Rhode Island. Using the Gibbs-Helmholtz equation to constrain the energy parameter in the van der Waals family of cubic equations of state (EOS), Dr. Lucia derives new expressions for the energy parameter in the Soave-RedlichKwong (SRK) equation for pure components and mixtures. These expressions contain the internal energy of departure, which functions as a natural bridge between the molecular length scale and the bulk phase length scale. Using coarsegrained values of the internal energy of departure from NTP Monte Carlo simulations, Lucia applies the multiscale Gibbs-Helmholtz constrained (GHC) EOS to determine densities of liquid $\mathrm{CO}_{2}$, water and mixtures of $\mathrm{CO}_{2}$, and water. Modeling results are compared to experimental liquid density data available in the open literature and show that these new GHC EOS provide significant improvements, matching experimental data to within $2 \%$.

The next article by Sakamoto et al. is a collaborative effort between the National Institute of Advanced Industrial for Science \& Technology (AIST) and Toho University in Japan and Los Alamos National Laboratory. The authors use hot water injection and depressurization laboratory experiments to study the effects of temperature, pressure, and permeability on methane hydrate dissociation. Numerical simulations and history matching using the Finite Element Heat and Mass Transfer reservoir simulator, FEHM, were conducted to help interpret the experimental results. The numerical simulations included the effects of a two-phase representation of methane hydrate and pore space and permeability as a function of porosity, hydrate saturation, and sand grain diameter. Results show that the experiments and numerical simulations are in good agreement.
The final paper in this special issue is a collaborative effort between the Chinese Academy of Sciences, Georgia Institute of Technology and the University of Maine led by Dr. Zuan Chen. In this work, Chen et al. look at the stability and deposition zones of natural gas hydrates on the north slope of the South China Sea. Seismic methods are used to study the gas hydrate stability zone (GHSZ) and gas hydrate deposition zone (GHDZ). The authors attempt to reconcile differences between prediction by GHSZ, GHDZ, and bottom simulating reflector (BSR) experiments at two separate sites in the north China Sea. The conclusions of the paper indicate that the presence of a stability zone is needed for deposition of hydrates and that numerical studies show reasonably good agreement between GHDZ and BSR.

\section{Angelo Lucia Jae W. Lee \\ Costas Tsouris}



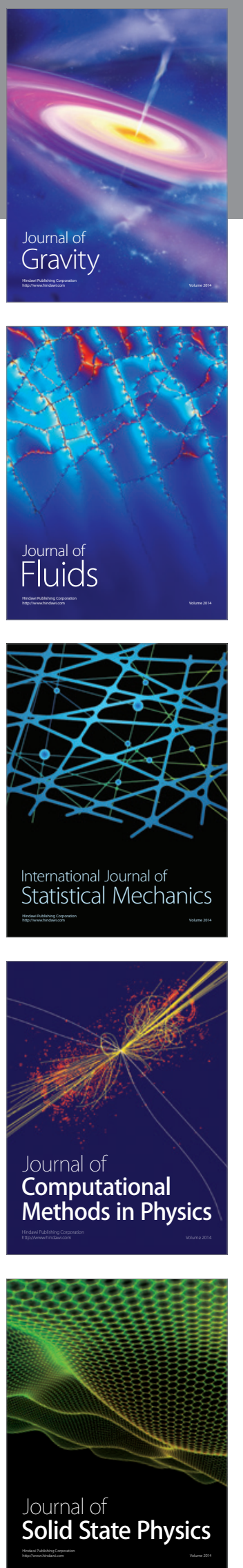

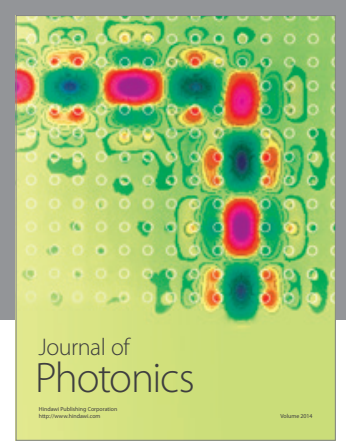

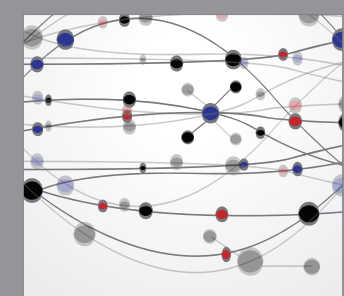

The Scientific World Journal
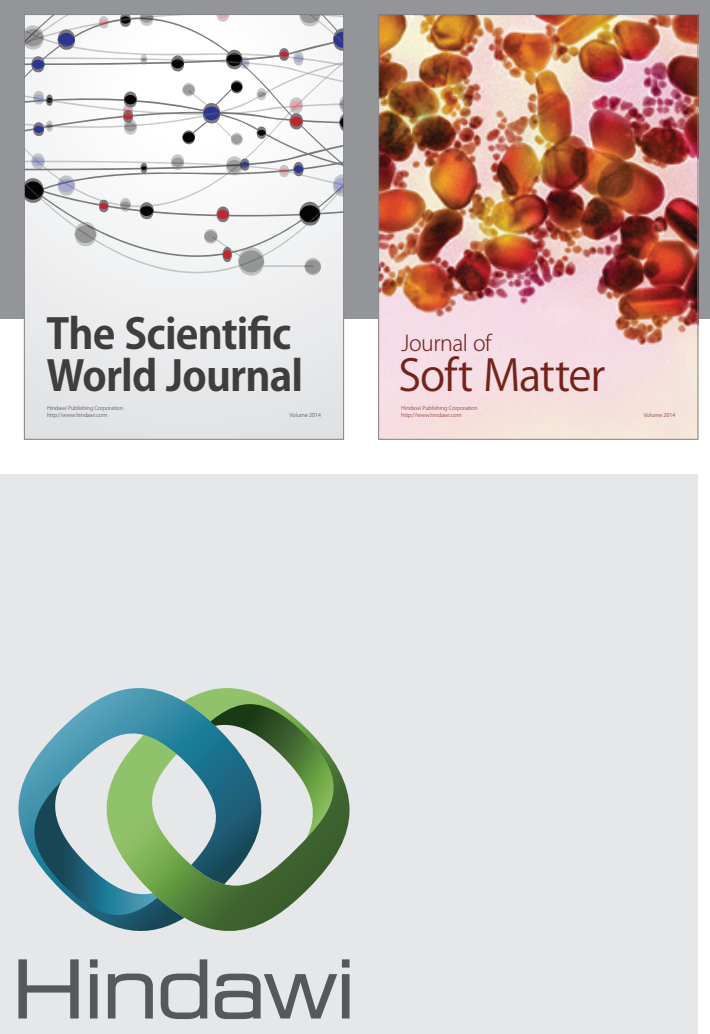

Submit your manuscripts at

http://www.hindawi.com
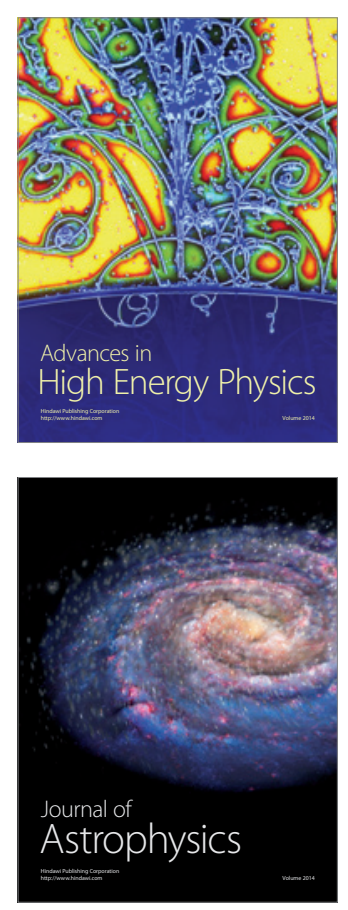
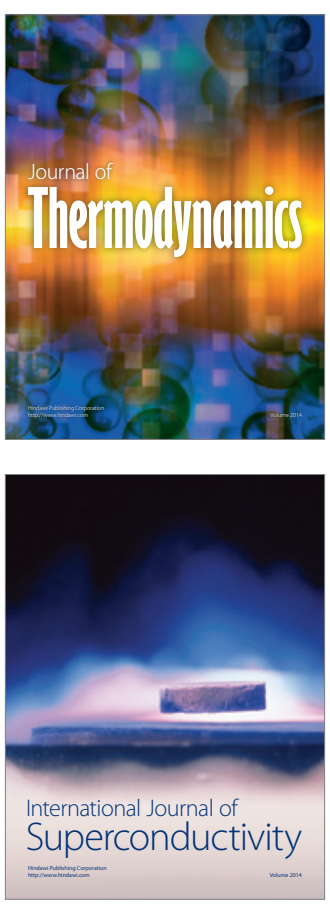
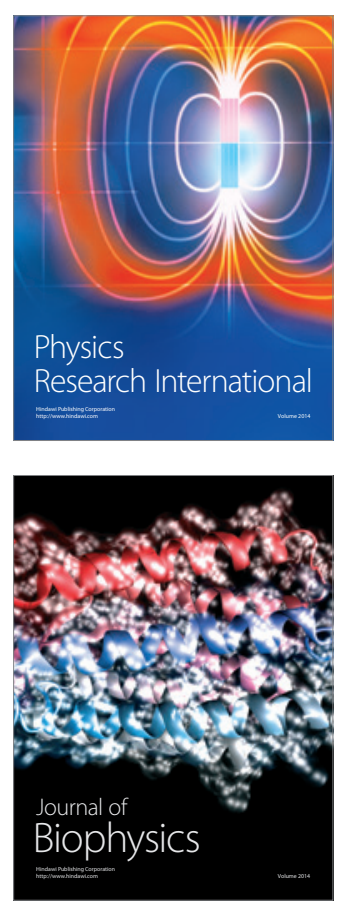
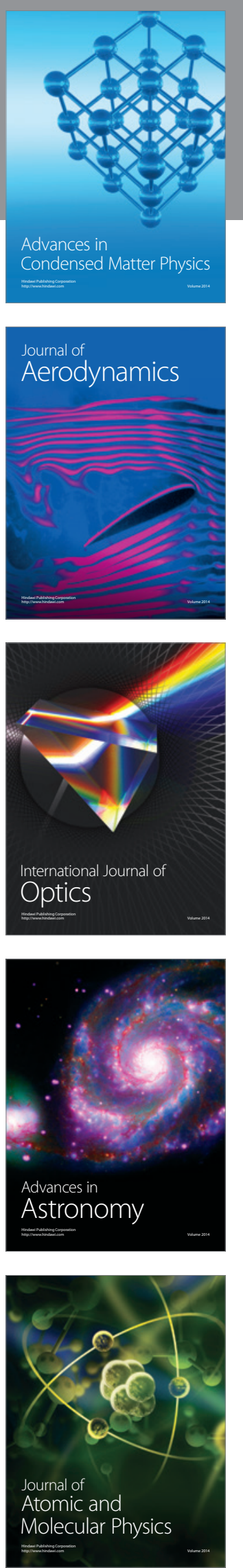